\title{
How Does It Feel to Clap Hands with a Robot?
}

\author{
Naomi T. Fitter ${ }^{1}\left[\right.$ Katherine J. Kuchenbecker $^{2}(\mathbb{D}$
}

Accepted: 12 October 2018 / Published online: 1 April 2019

(c) The Author(s) 2019

\begin{abstract}
Future robots may need lighthearted physical interaction capabilities to connect with people in meaningful ways. To begin exploring how users perceive playful human-robot hand-to-hand interaction, we conducted a study with 20 participants. Each user played simple hand-clapping games with the Rethink Robotics Baxter Research Robot during a 1-h-long session involving 24 randomly ordered conditions that varied in facial reactivity, physical reactivity, arm stiffness, and clapping tempo. Survey data and experiment recordings demonstrate that this interaction is viable: all users successfully completed the experiment and mentioned enjoying at least one game without prompting. Hand-clapping tempo was highly salient to users, and human-like robot errors were more widely accepted than mechanical errors. Furthermore, perceptions of Baxter varied in the following statistically significant ways: facial reactivity increased the robot's perceived pleasantness and energeticness; physical reactivity decreased pleasantness, energeticness, and dominance; higher arm stiffness increased safety and decreased dominance; and faster tempo increased energeticness and increased dominance. These findings can motivate and guide roboticists who want to design social-physical human-robot interactions.
\end{abstract}

Keywords Social-physical human-robot interaction $\cdot$ Social motor coordination $\cdot$ Robot affect

\section{Introduction}

Touch plays a crucial role in building emotional connections during human development and human-human interaction $[22,29]$. One natural touch mechanism is lighthearted handto-hand contact, from hand-clapping games between children to high fives among teammates or coworkers. Accordingly, as robots enter more human-populated environments and seek to connect with people, we anticipate that physical humanrobot hand-to-hand touch will be an important future source of mutual human-robot learning and bonding (Fig. 1). The widely varying kinesthetic and tactile aspects of these interactions result in a rich but complicated design space for

Electronic supplementary material The online version of this article (https://doi.org/10.1007/s12369-019-00542-x) contains

supplementary material, which is available to authorized users.

\section{Naomi T. Fitter \\ naomi.fitter@oregonstate.edu}

Katherine J. Kuchenbecker

kuchenbe@seas.upenn.edu; kjk@is.mpg.de

1 Collaborative Robotics and Intelligent Systems Institute, Oregon State University, Corvallis, OR 97331, USA

2 Haptic Intelligence Department, Max Planck Institute for Intelligent Systems, 70569 Stuttgart, Germany human-robot interaction (HRI) researchers. Previous socialphysical HRI (spHRI) studies have compared human-robot handshake behavior [1,3], object handover modes [8,18], and other human-robot tactile interactions further described in Sect. 2.

Our previous work details how we created a safe and capable hand-clapping robotic system by developing handclapping trajectory models and hand-contact detection strategies for the Rethink Robotics Baxter Robot [15], leading to the prototype used in the reported study. Here, the term "hand-clapping" refers to tempo-matching hand-to-hand contacts between two agents. In the style of early research steps in the aforementioned related literature, this article focuses on the thorough analysis of an initial human-robot experiment. After testing with one user in [15], we designed this experiment to expose many more users to our new handclapping robot behavior while investigating the implications of different robot contact acknowledgment modes, trajectory variability modes, and stiffness settings during interactions at various tempos.

Preliminary results from this study were reported in [16], looking only at users' overall qualitative reactions to the robot. This article augments these initial findings by examining the effects of the different tested conditions 


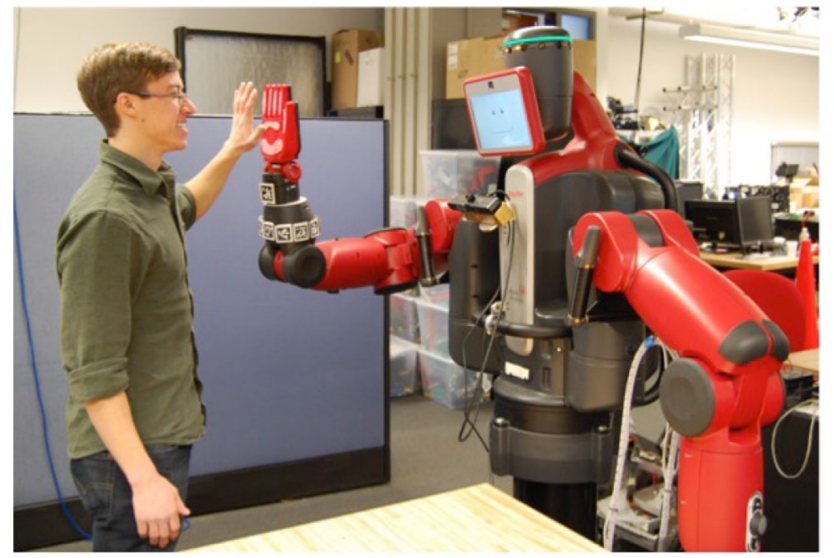

Fig. 1 The Baxter robot clapping hands with a person

and by analyzing additional data gathered throughout the investigation. This controlled initial experiment is an ideal springboard for understanding more complex future humanrobot hand-clapping game interactions like "Pat-a-cake" and "Slide." Other ongoing aspects of this work explore the usefulness of these hand-clapping games in interaction scenarios like promoting bonding before human-robot teamwork [13] and encouraging older adults to stay active by carrying out light exercise in their homes [12].

After summarizing related work (Sect. 2), this article describes how we tested our hand-clapping robot's abilities by conducting a user study (Sect. 3), extracting results from the experiment data (Sect. 4), considering the findings alongside the limitations of this research (Sect. 5), and summarizing overarching lessons (Sect. 6). We believe this research will aid and inform others who are interested in designing complex spHRI.

\section{Related Work}

Our work builds on two main areas: social robotics and physical human-robot interaction (pHRI). Combining these two fields creates the potential for emotional interaction augmented by a direct physical connection, as surveyed in [2]. Classic examples of effective social-physical robots are the robotic creature [33], the Huggable [30], and Paro [28]. In its various possible application areas, spHRI can serve functions from enhancing mental healthcare treatment [25] to helping a robot more fully comprehend human intention [19]. spHRI can even encourage communication from developmentally delayed children who otherwise would be unlikely to interact [26]. Our work looks to leverage the advantages of spHRI through human-robot hand clapping.

As a close parallel to our empirical investigation of handclapping HRI, research on human-robot handover tasks has illustrated that human users prefer minimum jerk trajectories along with other human-inspired robot motion controllers $[8,18]$. Human-robot handshake experiments have demonstrated ways to shape human-like robotic handshake algorithms using pure imitation or machine learning [3] and leveraging affective interaction design [1]. Although one study of robot touch in a medical setting indicates that users prefer pragmatic robot touch (washing the skin) to affective robot touch [7], we believe well designed playful robot touch in tempo-matching clapping games may be a more naturalfeeling interaction than a comforting pat from a robot in a mock medical setting. Like our work, all of these projects used empirical HRI experiments to compare subjective user responses to different robotic interaction modes.

Our experiment explores several interaction modes akin to those investigated in [1], a similar study of affective spHRI that focused on human-robot handshakes rather than hand clapping. In combination with investigations from the field of haptics, the affective handshake experiment inspires us to push the bounds of spHRI. Some haptics research delineates the perceived human- or robot-likeness of different robot control methods [7], including human motion data-driven strategies like the one used by our robot. Other work related to altering human-robot hand contact sensations includes studies on realistic contact rendering in virtual environments [11] and haptic illusions of softness [31]. This article pursues the understanding of haptic interactions with hand-clapping robots by investigating variables such as arm stiffness and system behavior during hand contact.

Although we believe our work is the first research focused on human-robot hand-clapping games, some past studies explored similar human-human activities. For example, investigations of human-human social motor coordination reveal that people are likely to engage in hand-clapping games throughout many stages of life and are skilled at synchronizing during this type of activity [27]. Study of jointly improvised motion lends similar insights about humanhuman synchronization and additionally reveals that expert improvisers may outperform others in some social motor coordinations tasks [24]. Additionally, [4] proposes a video game that uses electrodermal activity-sensing controllers to detect hand-to-hand contact between players for more enjoyable gameplay. In a similar way, [21] outlines the design and testing of an electrodermal activity-sensing wrist-worn watch designed to increase intimacy in a workplace environment.

HRI studies on other play applications also shaped our research. In educational environments, playful social robots have been shown to improve English language learning and help researchers estimate human friendship [20]. Qualitative evaluations helped researchers design affective understanding and reactions for the Haptic Creature [34]. Researchers have also observed natural human-robot play for social machine-learning applications using a small humanoid [9]. Practices in these previous studies indicate that we should 
collect qualitative data alongside quantitative subjective and sensor data in our initial hand-clapping HRI investigations.

\section{Methods}

We ran an experiment with the Rethink Robotics Baxter Research Robot to explore how human users perceive robotic hand-clapping playmates. Here, we were especially interested in how various robot interaction modes would affect impressions of robot safety and attributions of emotion to the robot. Accordingly, we gathered users' subjective responses to different styles of robot behavior while recording Baxter's sensor data and full-face video of the participant throughout the study. In this initial work, we selected a coarse sampling of conditions over the design space of possible robot interaction modes to gain a general understanding of this previously unexplored area. The Penn IRB approved all experimental procedures under protocol 823886 . The video included as supplementary material complements this Section's written explanation of the study methods.

\subsection{Robotic Platform}

We conducted this experiment using Baxter, a human-sized humanoid robot designed for interactive factory tasks. Baxter has a torso with two 7DOF arms, interchangeable grippers, and a panning head screen, as seen in Fig. 1. Although its intended use is pHRI in a factory setting, Baxter's humanoid design makes it an ideal candidate for spHRI. The robot's series elastic actuators, impact-absorbing shells, and fully backdrivable joints allow it to safely contact people in social scenarios. Further advantages stem from its standard Robot Operating System (ROS) framework and relatively affordable price $(\sim 25,000)$.

The commercially available Baxter parallel-jaw endeffectors proved unsuitable for hand-clapping interactions, so we developed custom 3D-printed non-articulating hands with inlaid silicone rubber contact pads, as shown in Fig. 2 [15]. Part files for our custom hands are available under a Creative Commons license at http://www.thingiverse.com/thing: 2286104. These end-effectors are average human-hand size to facilitate comfortable handclaps. The inlaid rubber protrudes beyond the hard plastic of the 3D-printed hand core to facilitate comfortable hand-to-end-effector contact that mimicks the sound of a human handclap.

\subsection{Experiment Setup}

Twenty participants (12 male and 8 female) enrolled in our study, gave informed consent, and successfully completed the experiment. Participants ranged from 19 to 38 years $(\mathrm{M}=27.2$ years, $\mathrm{SD}=5.0$ years $)$ and were mostly technical

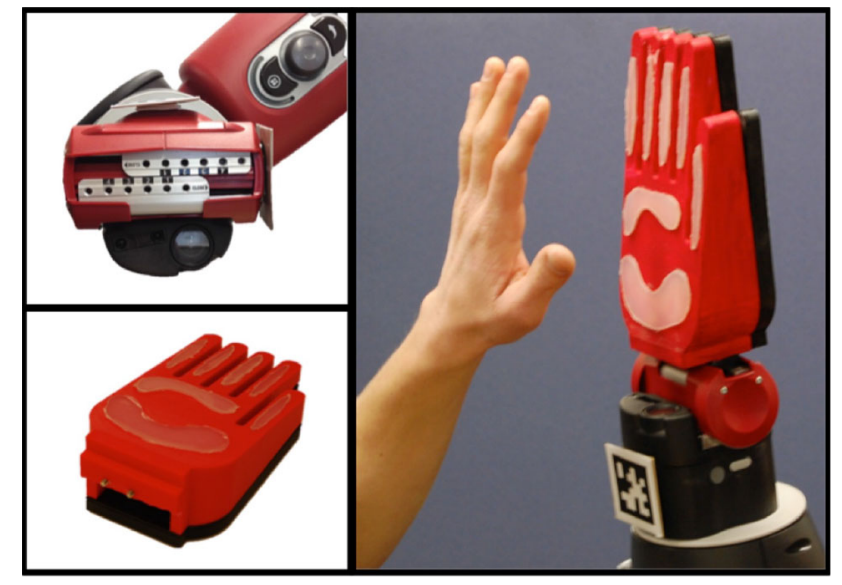

Fig. 2 Upper left: Baxter's built-in finger alignment rails with regularly spaced threaded holes. Lower left: our fabricated Baxter hand with M4 $\times 0.7$ screws compatible with the alignment rails. Right: the custom end-effector mounted on Baxter's arm

students (17 students, 1 educator, 1 postdoctoral researcher, 1 homemaker). Each person came to the lab for a single session that lasted about $1 \mathrm{~h}$. The participant stood facing Baxter throughout the experiment and engaged in repeated palm-to-palm contacts between their left hand and the robot's right end-effector, in the style of hand-clapping games. We designed this left-handed interaction to increase participant mental load during the experiment; all but one of the participants were right-handed. To keep participants as safe and comfortable as possible, the human-robot clapping location remained consistent and therefore predictable to users throughout the entire study. Because of this consistent clap location, it was easy for participants to step away from the claps or exit Baxter's workspace if they ever wanted a break for any reason. This experiment examined only one type of hand-clapping motion to increase the likelihood of successful execution by participants, but we are exploring other motions in ongoing work.

At the beginning of each session, the experimenter introduced Baxter to the participant and led the user in a practice human-human round of the hand-to-hand contact involved in experiment trials. Next, in each of 24 randomly ordered human-robot interaction trials, Baxter began to move its right end-effector along a hand-clapping trajectory at one of three possible hand-clapping frequencies $(60,110$, or 160 beats per minute (BPM)). We chose these specific tempos based on our past human-human hand-clapping study [15]. In that previous work, the fastest two tempos were so stressful that the temporal demand of keeping up dominated the interaction experience. Accordingly, we chose the three remaining tempos from that work that were neither boring nor unduly stressful. 
The participants started to play each trial's clapping game by contacting the robot after they believed they understood the robot's intended tempo and continued hand clapping for about $20 \mathrm{~s}$, after which Baxter returned to its starting pose. We asked participants to try to maintain the constant tempo set by the robot at the start of each interaction. A final humanrobot interaction trial allowed users to verbally select their favorite robot behavior mode and clap hands with the robot in that mode for as long as they desired.

\subsection{Conditions}

This experiment was designed to explore what people think about hand-clapping robots and quantify how different visual and haptic variables affect how users feel about the interaction. Our previous observations of human-human hand-clapping interactions focused on arm behaviors [15] and overall human emotional reactions [16]. Accordingly, to keep participants engaged and explore themes similar to the foci of our human-human observations, we designed different styles of Baxter facial animation, arm trajectory, and arm control. The following subsections detail how we designed each independent variable. To the best of our ability, we kept all other aspects of Baxter's behavior the same from trial to trial.

\subsubsection{Facial Animation}

Facial expressiveness can greatly affect human perception of a robot [14]. To begin exploring how differences in Baxter's face affect users, we designed facially reactive and facially nonreactive robot modes. It is important to note that this experiment considered facial responsiveness mainly as a way to signal human hand impact awareness and did not attempt to explore the large design space of Baxter facial expressiveness. Our work in [14] more rigorously assesses Baxter face image effects. Figure 3 illustrates the two expressions used to create the different robot face modes. These Baxter facial animation frames are consistent with several principles of robot face design discussed in [10]: wide facial proportions, more detail in the eyes than any other facial feature, and pres-

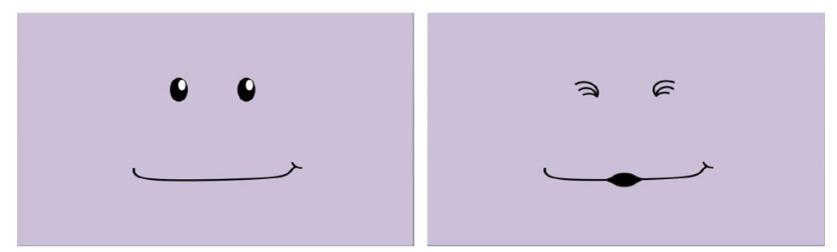

Fig. 3 Left: the default mildly positive Baxter facial expression. Right: the responsive facial expression used to animate the facially reactive robot ence of a mouth. The face design is intended to convey mild, but not uncanny, humanness.

In facially nonreactive mode, the robot's screen remained on the mildly positive face image at all times. In facially reactive mode, the mildly positive face was the default screen animation, and the responsive face appeared for $0.2 \mathrm{~s}$ after each detected hand contact. We identified hand impacts using the accelerometer in Baxter's moving hand [15]. Specifically, we thresholded the result of filtering the $\mathrm{x}$-axis of the right wrist accelerometer using a discrete-time first-order Butterworth high-pass filter with a cutoff frequency of $25 \mathrm{~Hz}$. The threshold was set to $4.0,5.0$, and $5.5 \mathrm{~m} / \mathrm{s}^{2}$ for the clapping tempos of 60,110 , and 160 BPM, respectively, to balance false positives with false negatives.

\subsubsection{Trajectory Variation}

A robot that can dynamically vary its hand trajectory based on human interaction behaviors may be perceived differently than a fixed-trajectory robot. Accordingly, another salient haptic interaction aspect to explore was the physical arm trajectory logic. Our previous investigation informed the employed strategies of hand-clapping robot movement and contact detection [15]. We found human motion to be generally sinusoidal and designed our robot to move with this same default behavior, as depicted in the top subplot of Fig. 4. Our previous work revealed a negative correlation between
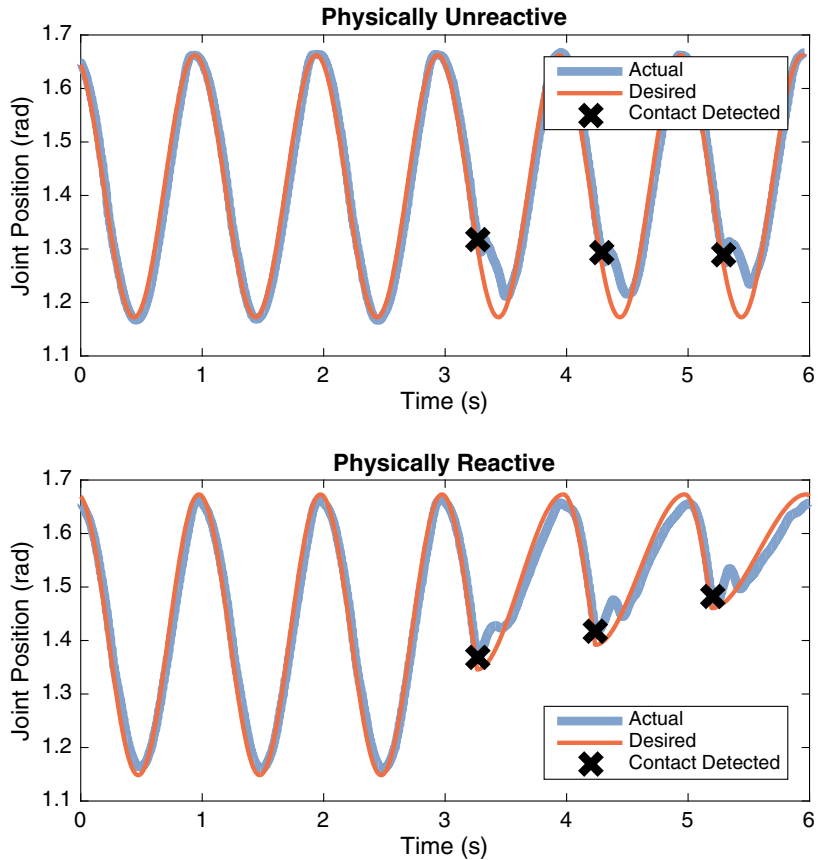

Fig. 4 Illustrative examples of physically unreactive versus physically reactive 60 BPM trajectories in Baxter W1 joint space. Each plot includes markers indicating hand contacts and the resulting trajectory after hand contact 
human hand-clapping frequency and amplitude [15], which we used here to determine appropriate amplitude values for each clapping frequency. Throughout experiment trials, only the wrist pitch (W1) joint of Baxter's right arm was commanded to move. The other joints were commanded to stay stationary, but their series elastic actuators are always soft, so the wrist motion caused passive natural-looking motion from the rest of the arm. The motion equation for the nonreactive mode of the robot's only active W1 joint was as follows, using variables for desired joint angle $\left(\theta_{d}\right)$, amplitude of motion $(A)$, frequency of hand contacts in $\mathrm{Hz}(f)$, time $(t)$, initial joint angle $\left(\theta_{\text {init }}\right)$, and a factor to keep handclap location the same regardless of tempo $\left(a_{\mathrm{comp}}\right)$ :

$\theta_{d}=A \sin (2 \pi f t+(\pi / 2))-A+\theta_{\text {init }}+a_{\text {comp }}$

The $\pi / 2$ shift causes Baxter's wrist to start at the farthest retreat location compared to the human partner.

In contrast, the robot's variable trajectory mode control strategy reacted dynamically. Whenever Baxter detected a handclap in this mode, its governing algorithm fit a cubic polynomial trajectory back to the extreme retreat location, as shown in the lower portion of Fig. 4. The start and end points of the trajectory were known because the start was simply the position and time of contact detection and the end was the farthest retreat location at a time calculated in order to maintain a constant robot hand-clapping frequency; the desired start and end velocities were zero because they represented instants of motion direction change. After achieving the retreat position, the robot returned to a sinusoidal approach trajectory until the next handclap. Later discussion will note that hand contacts very early in a motion cycle mandated a small cubic polynomial slope and thus slowed Baxter motion to maintain a constant clapping frequency.

\subsubsection{Stiffness}

Stiffness has been shown to influence how people perceive social-physical robots [1]. To explore the effects of robot arm stiffness in spHRI and keep users engaged with a diversity of interaction experiences, the final haptic condition that we varied was the stiffness of Baxter's arm throughout gameplay. We employed different proportional gains in Baxter's control law to accomplish varying stiffness. As developed previously [15], the overall time-domain control law of the robot's motion in this experiment is:

$\boldsymbol{\tau}_{\mathrm{cmd}}=\mathbf{K}_{d}\left(\dot{\boldsymbol{\theta}}_{d}-\dot{\boldsymbol{\theta}}\right)+\mathbf{K}_{p}\left(\boldsymbol{\theta}_{d}-\boldsymbol{\theta}\right)-\mathbf{K}_{f} \boldsymbol{\theta}_{d}+\boldsymbol{\tau}_{\mathrm{gc}}$

where $\boldsymbol{\tau}_{\mathrm{cmd}}$ is a vector of torques commanded to each Baxter arm motor, $\mathbf{K}_{d}$ is a diagonal matrix of derivative gains, $\boldsymbol{\theta}_{d}$ is a vector of desired arm joint angles, $\boldsymbol{\theta}$ is a vector of actual joint angles, $\mathbf{K}_{p}$ is a diagonal matrix of proportional gains,
$\mathbf{K}_{f}$ is a diagonal matrix of feedforward gains, and $\boldsymbol{\tau}_{\mathrm{gc}}$ is a vector of gravity compensation torques.

To maintain a consistent presented motion trajectory regardless of the trial conditions, we always used the same proportional gain $\left(30 \frac{\mathrm{Nm}}{\mathrm{rad}}\right)$ for the active $\mathrm{W} 1$ joint. For all other arm joints, we selected a lower proportional gain $\left(15 \frac{\mathrm{Nm}}{\mathrm{rad}}\right)$ to accomplish more compliant passive joint behavior and a higher proportional gain $\left(60 \frac{\mathrm{Nm}}{\mathrm{rad}}\right)$ for a more stiff arm joint behavior. In the equation above, $\mathbf{K}_{p}$ is the element that changes depending on the trial stiffness mode.

We investigated two levels of stiffness in our experimental design because of the clear role stiffness can play in strong and weak human-human hand-to-hand interaction; however, it is not certain that the trend between stiffness and user perception is monotonic. We thus discuss overall stiffness trends in this article using the assumption that the relationship between stiffness and participant perception is monotonic for the range of stiffness values tested. More evaluation would be needed to rigorously characterize this relationship.

\subsection{Hypotheses}

We selected the experimental conditions in anticipation that they would significantly affect the way users feel about clapping hands with Baxter. In particular, we hypothesized that:

- H1: Users will perceive a robot mode with responsive facial animation to be more pleasant than a facially unresponsive robot.

- H2: Users will perceive a variable trajectory robot to be less energetic, less dominant, and more safe than a robot that does not respond to their impact.

- H3: Users will perceive a stiffer robot to be more dominant and less safe than a robot with lower arm stiffness.

\subsection{Data Collection}

During every human-Baxter interaction, we recorded all available data from the robot's accelerometer, endpoint state, joint state, and face display ROS topics. We also recorded desired position, velocity, and feedforward torque at every timestep. The experiment was videotaped to enable retrospective review of the participants' facial expressions and any other notable experiment events.

Participants completed several surveys: (1) a robot evaluation survey after hearing introductory information about Baxter, (2) a hand-clapping game evaluation survey after practicing the game with the experimenter, (3) a subjective perception survey after each of the randomly ordered 20-s trials, (4) a concluding survey after the final unlimited interaction trial, and (5) a basic demographic survey after the conclud- 
Fig. 5 Adjectives used by participants in describing the hand-clapping experience, grouped by synonym. The size of the word reflects the frequency of use

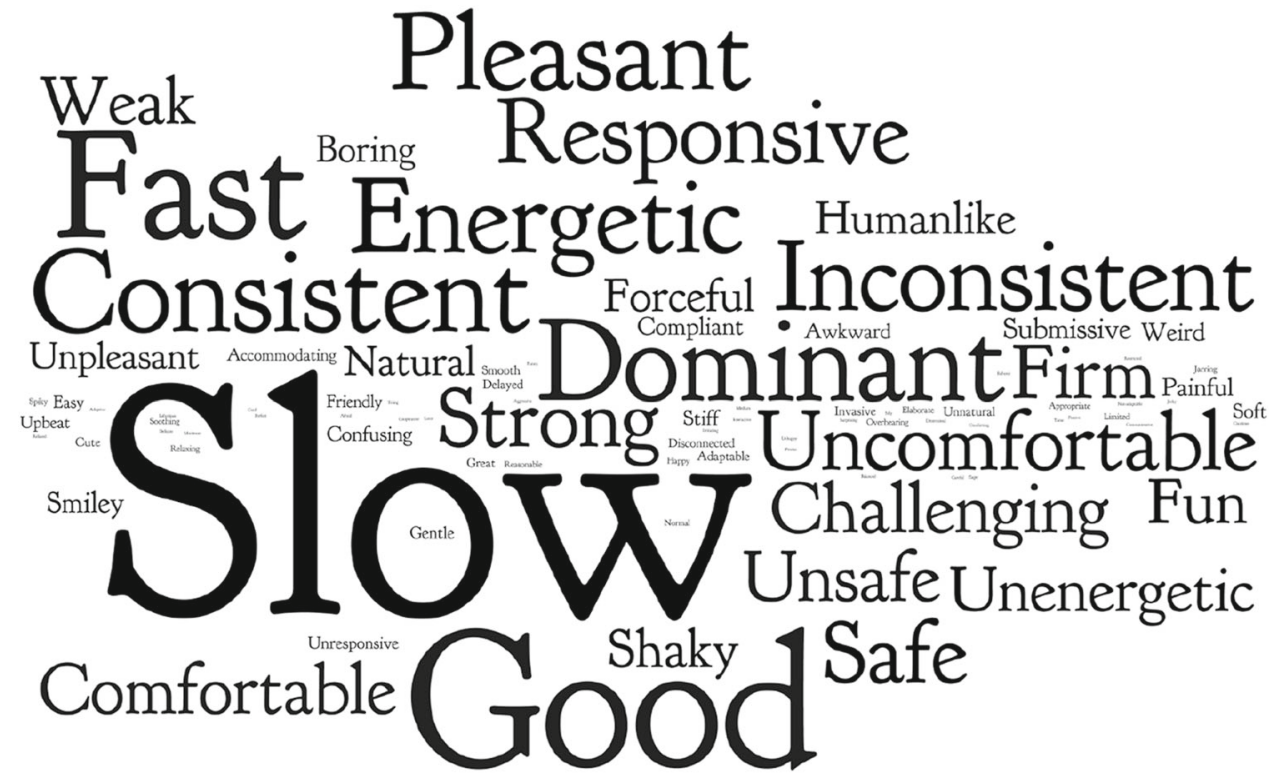

ing survey. The first two surveys involved only slider-type parametric questions. Survey (3) is analyzed extensively in Sect. 4, and thus the complete survey questions appear below:

- Please rate where the robot falls on this safety scale: (slider, "very unsafe to use" to "very safe to use")

- Please rate where the robot falls on this pleasingness scale: (slider, "very displeasing" to "very pleasing")

- Please rate where the robot falls on this energeticness scale: (slider, "not energetic" to "very energetic")

- Please rate where the robot falls on this dominance scale: (slider, "very submissive" to "very dominant")

- Why did you select these ratings? (extended text response)

The concluding survey contained a combination of slider and extended response questions.

Surveys for the experiment were carefully designed based on precedents in HRI research. The subjective trial perception survey leveraged the questionnaires used in [1] to evaluate robot engagingness based on the PAD (Pleasure, Arousal, Dominance) emotional state model [23] and also assessed perceived robot safety. Questionnaires (1), (2), and (4) were adapted from the Unified Theory of Acceptance and Use of Technology (UTAUT) and other metrics employed in [32] and [17].

\section{Results}

Qualitative and quantitative human user responses to interacting with Baxter, combined with recordings from robot sensors and experiment video footage, help us answer the question of how it feels to clap hands with a robot.
The results from each type of data are detailed in this Section.

\subsection{Qualitative Results}

We first analyzed the extended text responses to each Baxter experiment trial, as well as other experience-related metrics.

\subsubsection{Promising Aspects}

Study participants supplied us with a wealth of descriptive responses to the randomly ordered interaction trials. The word cloud in Fig. 5 presents all adjectives used by participants in the trial survey free-response field after interactions during which no errors occurred. (Errors are described in more detail in Sect. 4.1.2). Since each participant expressed a clear like and dislike of different trials, we find a balance of positive and negative descriptors in the word cloud. It is important to note that the large word "slow" always referred to the trial tempo, never the robot motion or responsiveness capabilities. Every user identified at least one interaction mode they enjoyed, as well as some that they did not like. Their preferences were not uniform. Some polarized opinions indicated that individualized interaction models may work best for distinct interactees, especially tempo-wise.

Users frequently remarked positively on Baxter's facial reactivity in survey responses and verbal commentary, labeling it with descriptors like "cute," "funny," "friendly," "pleasant," and "personable." Conversely, a lack of animation was often described as less pleasant, less sentient, and more robotic. This result was not completely universal; one user believed Baxter's responsive face to be an expression of pain, and another liked the facial anima- 
Table 1 Excerpts of each extended response entry linked to each observed type of experiment error

\begin{tabular}{|c|c|}
\hline Driving robot to stop & False positives \\
\hline $\begin{array}{l}\text { + I was trying to out-dominate [Baxter] } \\
+ \text { [We eventually found] a stable cycle } \\
+ \text { That felt the most natural so far } \\
+ \text { [Baxter] appeared dominant } \\
\text { - Responds too weakly to contact } \\
\text { - It made me feel unsure of [Baxter] } \\
\text { - [I felt] slightly less safe than before } \\
\text { - The game was less pleasing } \\
\text { - [This trial was] somewhat disconcerting } \\
\text { - Moving range of robot's hand too short } \\
\text { - A little too fast } \\
\text { - A bit too fast to be pleasing }\end{array}$ & $\begin{array}{l}+ \text { [Baxter] responded quickly } \\
\text { to making contact } \\
+ \text { I got the impression [Baxter] } \\
\text { was setting the pace } \\
+ \text { Good responsiveness, felt natural } \\
+ \text { Tempo was reasonable, [Baxter] } \\
\text { was not unnecessarily forceful } \\
\text { - The hand clapping felt odd } \\
- \text { The robot's hand was moving } \\
\text { up and down a lot }\end{array}$ \\
\hline "Jazz hands"-like oscillations & Human error \\
\hline $\begin{array}{l}\text { + At this speed, it feels energetic } \\
\text { - Something a little off - can't describe it } \\
\text { - [I had] the sense there was a malfunction } \\
\text { - I felt that something had gone wrong } \\
\text { - Shakiness was concerning and disruptive } \\
\text { - Palm-palm contact too long } \\
\text { - Not a fun rhythmic communication } \\
\text { - Motor started making disconcerting noise } \\
\text { - I think I liked this one the least so far } \\
\text { - [Baxter] seems afraid of clapping with me } \\
\text { - Trial was a little odd, hurt a little } \\
\text { - Felt unnatural for a clap } \\
\text { - Tempo got off, seemed schizophrenic } \\
\text { - [Robot] wrist vibrating more than usual } \\
\text { - [I] feel like the robot might not be safe }\end{array}$ & $\begin{array}{l}\text { + Hard to keep up, but still fun } \\
+ \text { [Robot response] feels good } \\
+ \text { It works well } \\
+ \text { [Baxter] adapted to the way I clap } \\
+ \text { [That made] a real clapping sound } \\
\text { - No consistent frequency of claps } \\
\text { - I didn't get the pattern } \\
\text { - Pattern doesn't repeat } \\
\text { - [Baxter] looked uncomfortable } \\
\text { - The tempo was pretty quick } \\
\text { - Tempo was faster than comfortable } \\
\text { - [Had to] bend my wrist uncomfortably } \\
\text { - [The hand] will jiggle sometimes } \\
\text { - It feels like I am hitting something } \\
\text { - A little too hard to keep [the] pace } \\
\text { - I thought the game was over, but then [Baxter] moved }\end{array}$ \\
\hline
\end{tabular}

The plus and minus signs indicate general positive or negative tone

tion at first but labeled it as "silly" near the end of the experiment.

In the final free interaction trial, users interacted with Baxter for eighteen or more seconds $(\mathrm{M}=43.95 \mathrm{~s}, \mathrm{SD}=$ $29.17 \mathrm{~s}$ ). This free interaction duration is approximately equal to or greater than the fixed length trial interactions. Based only on brief verbal descriptions of the options, without linking the words to particular observed behaviors, users selected the following customized robot behaviors for their free interaction trials: 20/20 selected facially animated, 15/20 selected variable trajectory, and 9/20 selected high stiffness. For tempo, 1 selected 60BPM, 14 selected 110 BPM, and 5 selected 160 BPM. This variety of choices indicates that we aptly designed diverse robot interaction behaviors.

\subsubsection{Error Accounts}

Although eight of the 20 study participants reported never perceiving Baxter to have made any error, other participants encountered some challenges and errors during the 500 total trials of the experiment, as summarized in Table 1. In the fastest-tempo physically reactive mode, seven participants responded to Baxter's retreat reaction by sometimes contacting the robot's end-effector earlier and earlier in its motion cycle (12 total trials). Because the retreat motion was capped at the far retreat position, this user strategy resulted in a decrease in robot motion amplitude and, in some extreme cases, momentary periods of Baxter stillness, which sometimes perplexed users. Reactions to the robot stopping varied from satisfaction and acceptance to displeasure and distrust. 
CC1: People would be impressed if I had such a robot.

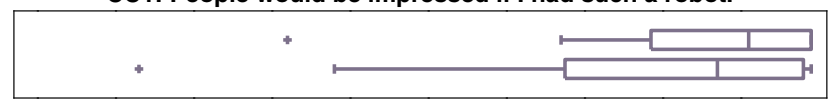

CC2: Robots are nice to work with.

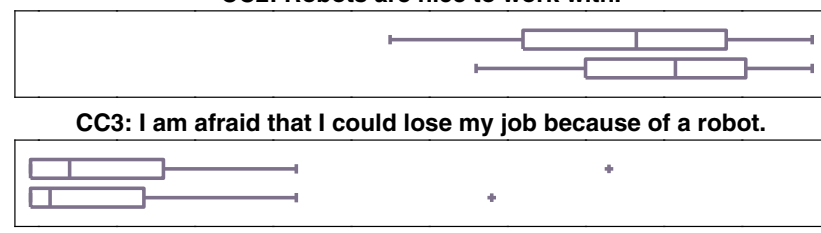

GR1: I like the presence of the robot.

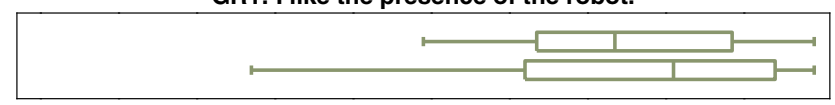

GR2: I could do activities with this robot.

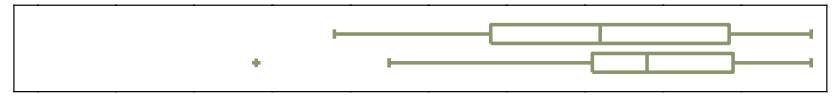

GR3: I feel threatened by the robot.

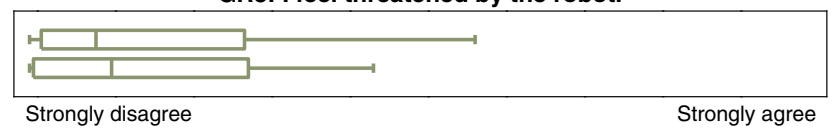

Fig. 6 Survey responses to analogous pairings of robot-related questions on the initial robot evaluation and concluding surveys. In each plot, the top box plot represents the participant responses to the question on the robot evaluation survey, and the bottom box plot represents the responses on the concluding survey. The center box line represents

Next, thresholding the accelerometer data produced six observable false positive hand contact detections, most often in trials with a physically responsive trajectory and low arm stiffness. In these cases, vibrations from the robot's own movement made it think the user had contacted its hand when they had not. This error bothered some users, but not others. Perhaps because this error is a natural-feeling and humanlike mistake, only two of the false positive incidents resulted in negative user commentary.

A final robot behavior problem stemmed from controller gains; five participants were able to exert enough axial torque on Baxter's end-effector to cause surprising, "jazz hands"like oscillations in Baxter's wrist roll (W2) joint motion (15 total trials). Users disliked this error type; the mechanical "jazz hands" behavior seemed unusual and was described as almost unanimously problematic in survey comments.

Human users also made sixteen errors throughout the experiment, namely failing to match Baxter's fastest clapping frequency or misunderstanding Baxter's slower frequencies. Users had a balance of positive and negative responses to these trials as well. The various human errors may be avoided in the future with better experiment design.

\subsubsection{Overall Impressions}

Despite these occasional errors, participants' opinions of Baxter did not change significantly in a positive or negative way over the course of the experiment, as illustrated in
SE1: I would feel comfortable interacting with the robot.

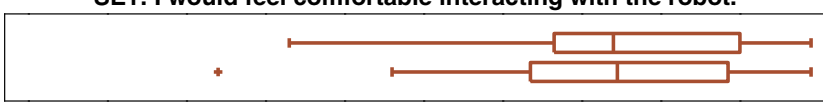

SE2: I could work with the robot if someone helped me.

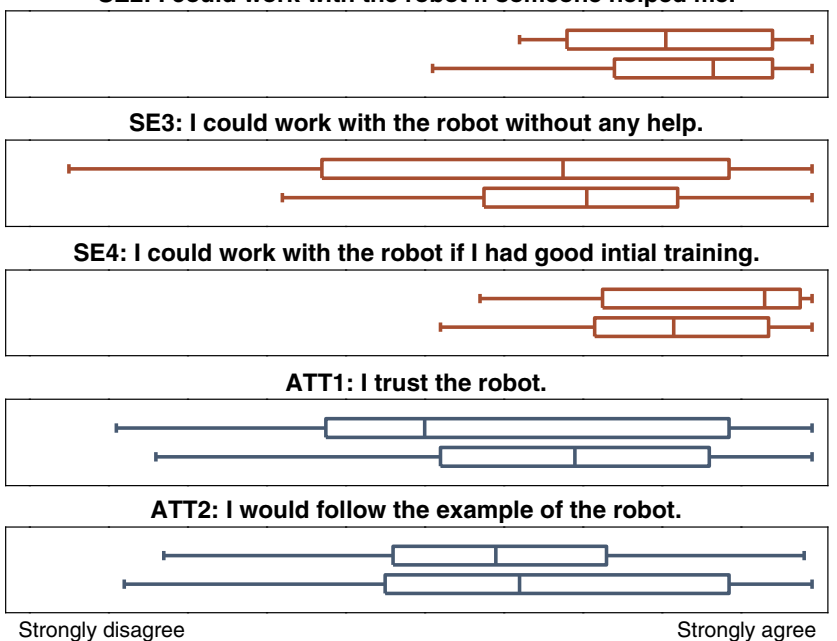

the median, and the box edges are the 25 th and 75 th percentiles. The whiskers show the range up to 1.5 times the interquartile range, and outliers are marked with a "+". The question coding abbreviations stand for cultural context (CC), forms of grouping (GR), self-efficacy from UTAUT model (SE), and attachment (ATT)

Fig. 6. The statements in the subplot titles appear exactly as shown to participants; no additional explanations were provided. Paired t-tests reveal no significant difference between each survey question's pair of before and after responses (all $p>0.23$ ). A comparison of before and after survey responses grouped by general category (cultural context, forms of grouping, self efficacy, and attachment) similarly yielded no statistically significant differences (all $p>0.27$ ), although the median response in all categories except selfefficacy increased in the post-interaction survey.

We also see some consensus in positive and negative remarks in the concluding survey essay responses. Table 2 illustrates a balanced set of experiment feedback, including praise of interaction modes alongside critiques indicating that we need more customizability in the robot behaviors. In a final essay question, nineteen of the 20 users identified a personal interest in interacting with Baxter in some way. Use ideas included experiment-like tasks such as playing more complicated hand-clapping games, collaboratively manipulating objects, and doing arm exercises; chore-like tasks such as cooking, washing dishes, doing laundry, and cleaning bathrooms; and social tasks like performing music/dance, playing sports/board games, drinking beer, and socializing.

\subsection{Trial Survey Results}

We next focused on analyzing the subjective trial questionnaire results to understand how specific interaction factors 
Table 2 Responses occurring at least twice in concluding survey essay responses

\begin{tabular}{ll}
\hline Positive/enjoyable aspects & Negative/difficult aspects \\
\hline Getting to engage with Baxter (9) & Interaction too long for healthy adults (6) \\
Baxter's facial expressions (6) & Hard to get tempo right in fast trials (6) \\
Scientific aspects of experience (6) & Any robot errors/inconsistencies (4) \\
Quick and responsive robot behaviors (4) & Robot too short (4) \\
Getting to choose final trial conditions (3) & Some settings uncomfortable (3) \\
Predictable robot behaviors (2) & Interaction needs more motion variety (3) \\
Exploring different interaction modes (2) & Hand got sore/tired (3) \\
Fun/bonding aspects of interaction (2) & Baxter not as skilled as a human (2) \\
\hline
\end{tabular}

The numbers indicate response frequency
Table 3 The $p$ values returned from rANOVAs on the safety and affect ratings

\begin{tabular}{|l|c|c|c|c|}
\hline \multicolumn{1}{|c|}{ Metric } & $\begin{array}{c}\text { Facial } \\
\text { Reactivity }\end{array}$ & $\begin{array}{c}\text { Physical } \\
\text { Reactivity }\end{array}$ & Stiffness & Tempo \\
\hline Safety & 0.0675 & 0.2311 & 0.0164 & 0.2683 \\
\hline Pleasantness & 0.0069 & 0.0151 & 0.1115 & 0.6012 \\
\hline Energeticness & 0.0235 & 0.0013 & 0.1331 & $<0.0001$ \\
\hline Dominance & 0.7230 & 0.0123 & 0.0145 & $<0.0001$ \\
\hline
\end{tabular}

Positive changes from off/off/low/slow to on/on/high/fast are highlighted in light gray. Negative changes are highlighted in dark gray

affected the hand-clapping gameplay experience. Our main tool throughout this process was repeated measures analysis of variance (rANOVA), a statistical method that enables us to determine whether the presented visual and haptic conditions affected user perceptions of Baxter's pleasantness, energeticness, dominance, and safety.

The intended within-subject factors for our rANOVA were presence or absence of facial reactivity, presence or absence of physical reactivity, and low or high arm stiffness (a $2 \times 2 \times 2$ design space). While testing for other significant conditions, we discovered that tempo was also highly influential, although the three different tempos used in the experiment were originally intended to serve as three repetitions of each experimental condition. After making this discovery, we included tempo as a within-subject factor, leading us to carry out a $2 \times 2 \times 2 \times 3$ four-factor rANOVA.

The overall rANOVA statistical results appear in Table 3, and the box plots in Fig. 7 and 8 illustrate the different data partitions that result from sorting data by each within-subject factor. We fill in any boxes representing a pairing of data with significant differences, as determined by examining $p$ values from our rANOVA tests at an $\alpha=0.05$ significance level. We report for significant effects using $\eta^{2}$. These differences are referenced throughout the following discussion of condition results, which includes the testing of our hypotheses.
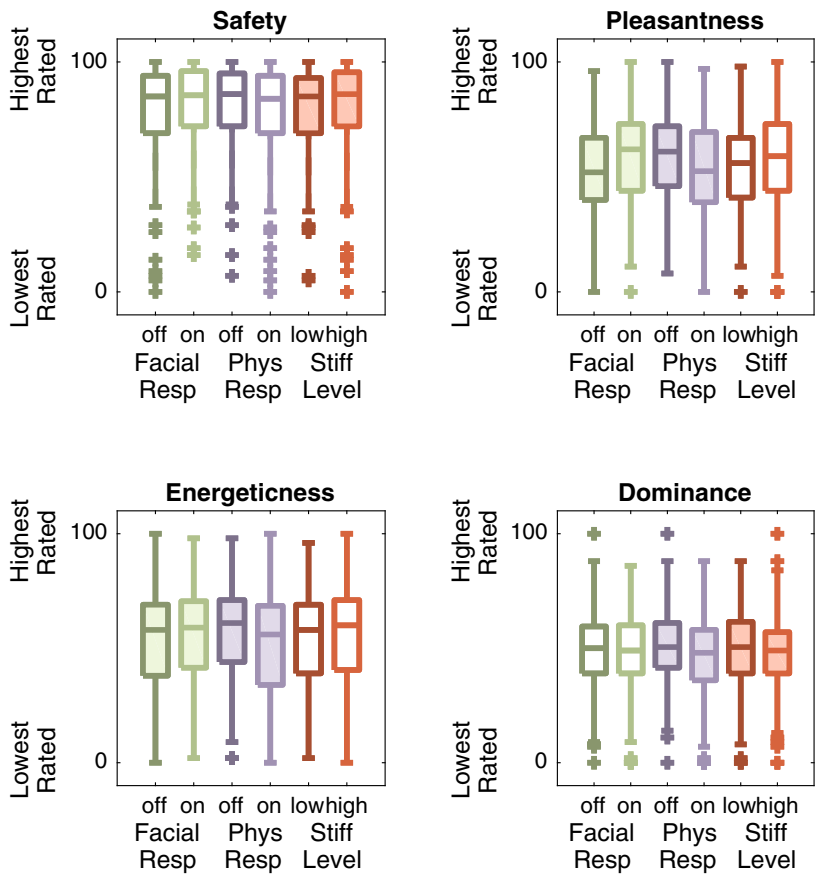

Fig. 7 Participant responses to trial survey questions, separated by all conditions except tempo. The center box line represents the median, and box edges are the 25th and 75th percentiles. The whiskers show the range up to 1.5 times the interquartile range, and outliers are marked with a "+"

\subsubsection{Facial Animation}

Facial reactivity had significant positive effects on two ratings (Fig. 7). As predicted in H1, participants did perceive a facially responsive robot as more pleasant than a facially static one $\left(\mathrm{F}(1,19)=9.17, p=0.0069, \eta^{2}=0.0183\right)$. Users also saw the facially reactive robot as more energetic $\left(\mathrm{F}(1,19)=6.06, p=0.0235, \eta^{2}=0.0036\right)$. Facial reactivity did not affect perceived safety or dominance. 


\subsubsection{Trajectory Variation}

User impressions of physical reactiveness significantly affected three ratings, meeting some of our expectations and overturning others (Fig. 7). We did not expect this condition to affect perceived pleasantness of the robot, but users reported that the variable trajectory robot was less pleasant than a robot that maintains the same trajectory regardless of perceived hand impacts $(\mathrm{F}(1,19)=7.14, p=0.0151$, $\left.\eta^{2}=0.0234\right)$. Conversely, we correctly predicted in $\mathbf{H 2}$ that a physically reactive robot would appear less energetic $\left(\mathrm{F}(1,19)=14.12, p=0.0013, \eta^{2}=0.0223\right)$ and less dominant $\left(\mathrm{F}(1,19)=7.65, p=0.0123, \eta^{2}=0.0212\right)$. Trajectory variation did not affect perceived robot safety.

\subsubsection{Stiffness}

The results contradicted our predictions of stiffness effects on user perception (Fig. 7). Although we hypothesized in H3 that a stiffer robot would appear less safe and more dominant, participant responses revealed that users perceived a stiffer robot to be more safe $(\mathrm{F}(1,19)=6.93, p=0.0164$, $\left.\eta^{2}=0.0043\right)$ and less dominant $(\mathrm{F}(1,19)=7.24, p=$ $\left.0.0145, \eta^{2}=0.0055\right)$. Stiffness did not affect pleasantness or energeticness.

\subsubsection{Tempo}

As noted previously, tempo had significant effects on the results, evoking some of the strongest trends in user response (Fig. 8). Participants found robots interacting at different tempos to have significantly different energy levels $\left(\mathrm{F}(2,38)=144.01, p<0.0001, \eta^{2}=0.4215\right)$ and dominance levels $\left(\mathrm{F}(2,38)=24.86, p<0.0001, \eta^{2}=0.1340\right)$. A post-hoc Tukey multiple comparisons test shows that at each increasing tempo, Baxter seems significantly more energetic and more dominant. Tempo did not affect robot safety or pleasantness.

\subsection{Robot Recording Results}

Although the self-reported participant responses were informative, we were also interested in examining some more objective measures to see if they could help elucidate what happened during each trial. The data recorded from Baxter's wrist accelerometer in particular allowed us to examine errors between the actual and intended tempo during each trial (a metric reflecting synchronization) and the peak contact acceleration during each hand impact (a proxy for peak contact force). As in our analyses of the trial survey responses, we use a $2 \times 2 \times 2 \times 3$ four-factor rANOVA at an $\alpha=0.05$ significance level for these tests. The overall rANOVA statistical results appear in Table 4 , and the box
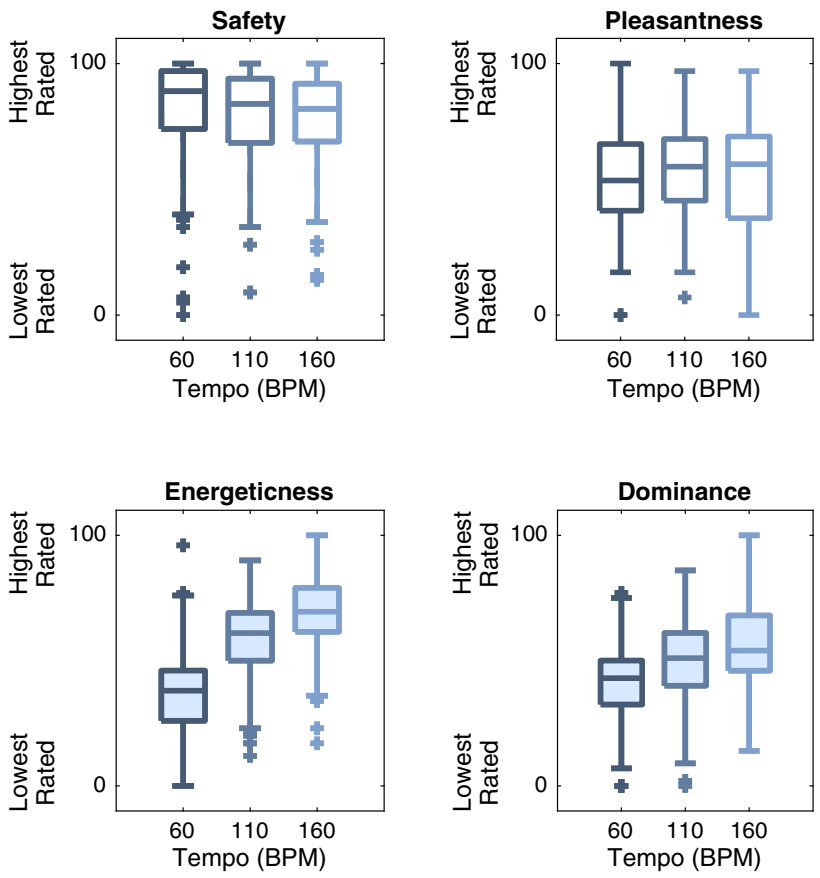

Fig. 8 Participant responses to trial survey questions, separated by tempo condition. The center box line represents the median, and box edges are the 25 th and 75 th percentiles. The whiskers show the range up to 1.5 times the interquartile range, and outliers are marked with a "+"

Table 4 The $p$ values returned by our objective measure rANOVAs

\begin{tabular}{|l|c|c|c|c|}
\hline \multicolumn{1}{|c|}{ Metric } & $\begin{array}{c}\text { Facial } \\
\text { Reactivity }\end{array}$ & $\begin{array}{c}\text { Physical } \\
\text { Reactivity }\end{array}$ & Stiffness & Tempo \\
\hline Timing Error MSE & 0.1883 & 0.9784 & 0.0265 & 0.1483 \\
\hline Timing Error Median & 0.7969 & 0.0273 & 0.7525 & $<0.0001$ \\
\hline Timing Error SD & 0.2213 & 0.0557 & 0.0022 & 0.0681 \\
\hline Contact Accel Median & 0.4614 & 0.0137 & 0.1655 & $<0.0001$ \\
\hline Contact Accel SD & 0.8378 & 0.7800 & 0.0137 & 0.1452 \\
\hline
\end{tabular}

Positive changes from off/off/low/slow to on/on/high/fast are highlighted in light gray. Negative changes are highlighted in dark gray

plots in Figs. 9 and 10 illustrate the different data partitions that result from sorting recorded data by each within-subject factor.

\subsubsection{Synchronization Analysis}

Synchronization metrics can help evaluate how well Baxter and the experiment participant were working together to accomplish the target clapping tempo during each interaction trial, revealing additional interactional effects of the different hand-clapping conditions. Accordingly, we calculated the error between each trial's target inter-clap time interval (known from the trial tempo) and the actual intervals (found by computing the difference between recorded times of hand contact) for each experiment trial. To encompass a variety of descriptive information, we extracted the mean squared error 

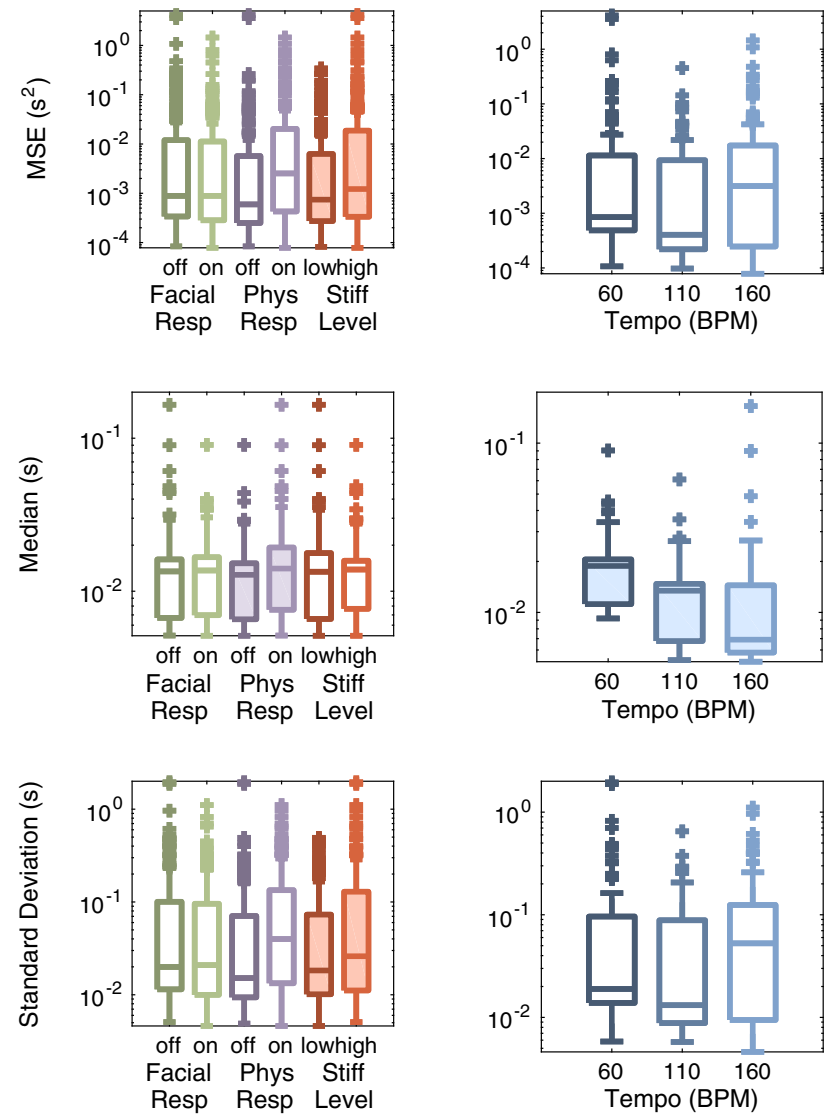

Fig. 9 Comparison of inter-clap interval timing errors for different trial conditions

(MSE) in timing for each trial and also gathered the statistical measures of median and standard deviation in timing errors per trial.

The rANOVA on each timing error metric reveals that synchrony varied over some trial conditions (Fig. 9). Facial responsiveness did not affect trial timing. Physical responsiveness resulted in a greater median timing error $(\mathrm{F}(1,19)=$ 5.72, $\left.p=0.0273, \eta^{2}=0.0137\right)$. A stiffer robot arm led to a greater timing $\operatorname{MSE}(\mathrm{F}(1,19)=5.79, p=0.0265$, $\left.\eta^{2}=0.0109\right)$ and also a greater standard deviation of clap timing errors $\left(\mathrm{F}(1,19)=12.49, p=0.0022, \eta^{2}=0.0218\right)$. Tempo also affected clap timing errors; median timing error dropped for higher tempos $(\mathrm{F}(2,38)=11.23, p<0.0001$, $\left.\eta^{2}=0.0617\right)$. A post-hoc Tukey multiple comparisons test shows that the median timing error value was significantly lower for the 110 and 160 BPM tempos than the 60 BPM tempo.

\subsubsection{Contact Accelerations}

Differences in contact acceleration could indicate user comfort with the robot, but forces that are too high could become painful after prolonged exposure. Thus, observing how hard
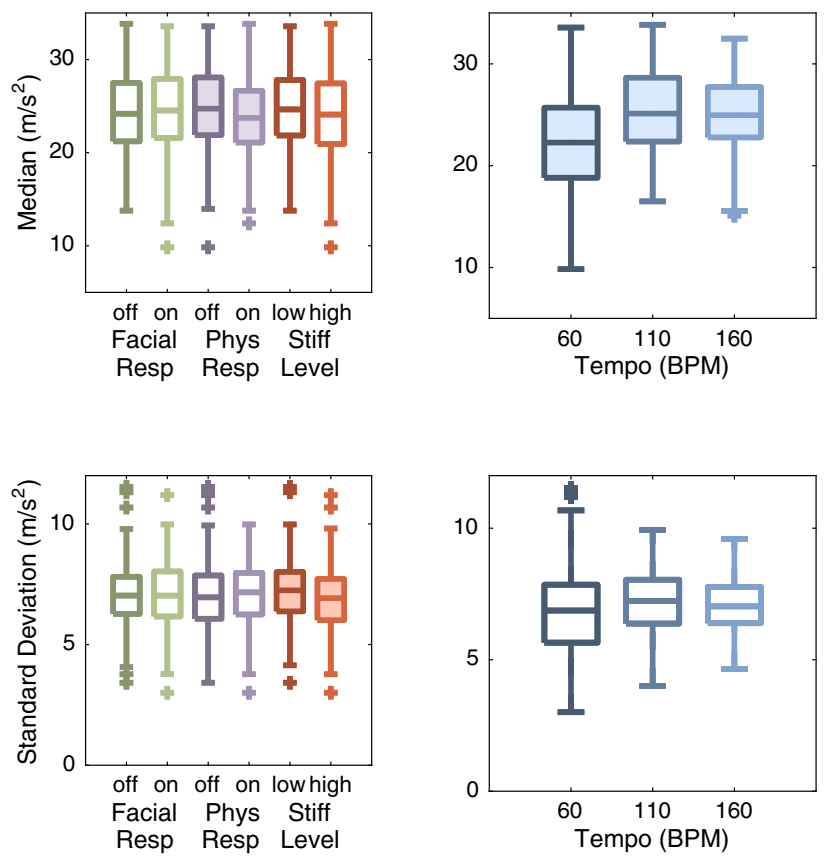

Fig. 10 Illustration of acceleration at hand contact over different types of trial

participants clapped with Baxter under different conditions can help us compare interaction modes. We located the times of hand contact in experiment recordings using the same accelerometer filtering technique described in Sect. 3.3. The acceleration readings at all the times of hand impact were extracted from each experimental trial recording. We then computed the median and standard deviation of the peak accelerations from each trial.

A rANOVA on each contact acceleration metric shows some statistically significant differences (Fig. 10). Facial responsiveness did not affect hand contact acceleration. Physically reactive trials were accompanied by a decreased median contact acceleration $(\mathrm{F}(1,19)=7.38, p=0.0137$, $\eta^{2}=0.0086$ ). Trials with a stiffer robot arm displayed a decreased standard deviation in peak contact acceleration $\left(\mathrm{F}(1,19)=7.37, p=0.0137, \eta^{2}=0.0116\right)$. Trials with faster clapping tempos showed a higher median contact acceleration $\left(\mathrm{F}(2,38)=16.58, p<0.0001, \eta^{2}=0.0862\right)$. A post-hoc Tukey multiple comparisons test shows that the median acceleration for the 110 and 160 BPM tempos was significantly higher than for the 60 BPM tempo.

\subsection{Video Recording Results}

The main user behavior that we quantified via the experiment videos was the number of times the user looked at Baxter's face throughout the trials. These glances may be a good proxy for social referencing, a phenomenon that occurs when one person looks to another person during an unfamil- 

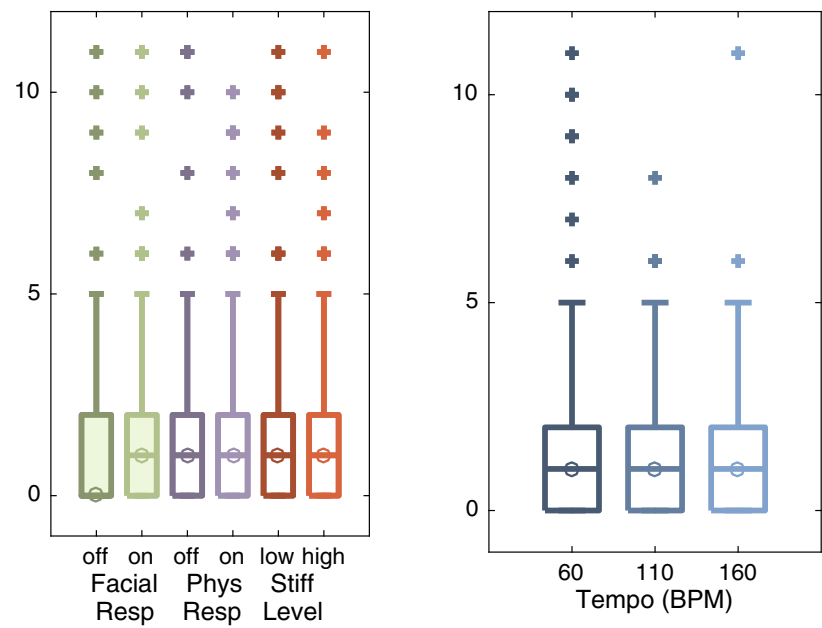

Fig. 11 Visualization of the number of face referencing occurrences between the participant and Baxter during each trial under different conditions. The circles indicate the positions of the medians

iar situation for guidance or reassurance [6]. Face referencing occurred in different ways from user to user, but the overall trends can help us understand the influence of Baxter's face. An experienced rater watched all of the experiment footage videos and tallied every event where the participant looked at the robot's face. Figure 11 illustrates the distribution of this occurrence over different trial conditions. A statistical test reveals that only the facial responsiveness condition affects the facial reference count. Participants looked at Baxter's face more often when it was facially animated $(\mathrm{F}(1,18)>1000$, $p<0.0001, \eta^{2}=0.9999$ ).

Although self-reported responses to the robot and handclapping experience were fairly positive throughout the study, we also sought a more objective measure of participant experience through the study videos. We analyzed the participant emotions exhibited in each video frame using the facial Action Unit (AU) extraction abilities of the OpenFace tool [5]. Figure 12 illustrates the total time that each participant exhibited all of the AUs indicative of each of five main human emotions: happiness, sadness, surprise, fear, and anger. The high occurrence of happiness supports that participants enjoyed the study. A rANOVA on facial expression durations across emotion revealed that there is a significant difference in how long participants displayed different types of emotion $\left(\mathrm{F}(4,72)=18.60, p<0.0001, \eta^{2}=0.4542\right)$. A post-hoc Tukey multiple comparisons test revealed that participants displayed happy expressions significantly more than any other expression.

\section{Discussion}

The results of this human-robot experiment begin to answer the question of how it feels for human users to clap hands

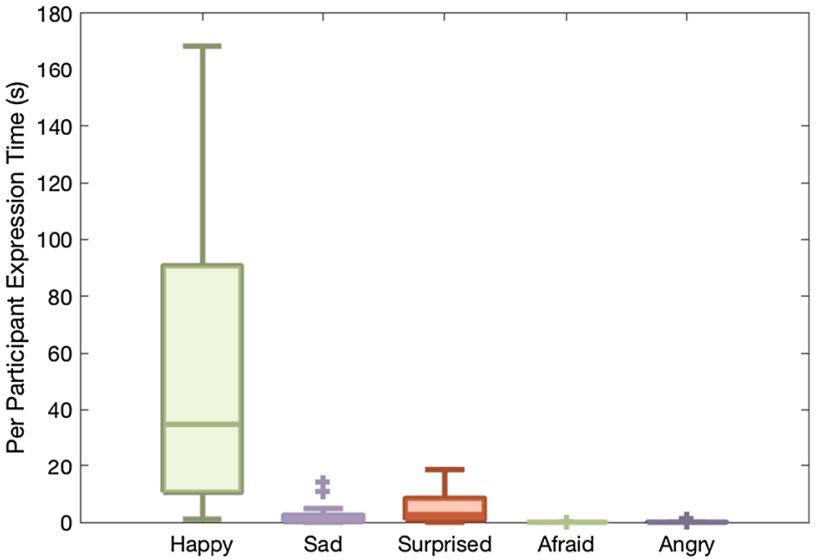

Fig. 12 Amount of time each participant displayed the analyzed core human emotions

with Baxter. Our analyses focused especially on the effects of Baxter's facial responsiveness, physical responsiveness, and stiffness during interaction trials. Although the results confirmed some of our hypotheses, other expectations were incorrect, and the data revealed several unpredicted effects.

User impressions of Baxter, as indicated by the responses to the UTAUT-inspired survey, did not change significantly from before to after the experiment. We interpret this consistency to signify that although this version of our handclapping robot made some errors, it is worth continuing to develop improved system behaviors. The fact that we purposely tested some "bad" robot modes may have also contributed to the lack of change between the before and after UTAUT responses.

Our findings upheld H1's prediction that a facially animated robot would appear more pleasant. The additional effects of facial responsiveness outside of those predicted by H1 appear logical; increased energeticness of a facially animated robot may stem from extra dynamism and appearance of social intelligence. When asked if Baxter's facial expression changed throughout the experiment, three of the 20 participants reported never noticing a change. Nevertheless, the statistical results with or without these three individuals remain the same. Users also glanced more at the responsive face.

For the physically responsive robot, $\mathbf{H 2}$ correctly forecasted a decrease in energeticness and dominance ratings. We believe the negative pleasantness ratings for the physically reactive robot arose from some confusion about how to interact with this robot mode. In the fastest-tempo physically reactive mode, several participants responded to Baxter's retreat reaction by contacting the robot's end-effector earlier and earlier in its motion cycle, which caused slow motion and some momentary periods of Baxter stillness. Some users also felt confusion due to the unannounced switch from robot leadership (in defining the interaction tempo initially) to 
human leadership (in defining contact position during physically reactive trials). Some users seemed perplexed as a result, so the overall safety ratings did not match the positive safety trend predicted by $\mathbf{H} \mathbf{2}$.

The stiffness results, all contrary to $\mathbf{H 3}$, most likely arose from the fact that a higher proportional gain in the controller causes the robot to more closely follow its programmed trajectory. Baxter movement at low stiffnesses was more likely to vary, and accordingly, result in the appearance of dominant volition and less safety. Similarly, mechanical errors arising from wrist roll joint stiffness were less likely to be forgiven than other more human-like errors that occurred in the experiment. People seem to prefer a robot that moves predictably.

Tempo results all appear logical: a faster robot is literally more energetic and harder to stop. As similarly seen in other emergent HRI work [35], the timing of Baxter's motion can strongly influence how energetic and dominant it seems. The contact acceleration of handclaps also increases significantly as tempo increases. Although trials at different tempos were originally intended to be repetitions of conditions, some of the strongest user rating and robot recording differences came from tempo differences.

Other informal observations throughout the experiment indicated positive aspects of the designed robotic system and experimental interaction. For example, all enrolled participants were comfortable enough with Baxter's appearance to consent to the experiment. The experimental activity was also sufficiently captivating so that all users completed the full experiment, even in this extremely repetitive use case. Although the robot made some mistakes, safety ratings of the robot were still generally high. When asked if the robot made any type of mistake throughout the entire experiment, eight of the 20 participants believed the robot's behavior to be perfect. Additionally, users enjoyed Baxter's facial expressions and often attributed inventive, non-existent sensing intelligences to the robot.

In contrast, the study design had some shortcomings. The user population in this study was fairly small and was composed of mostly young technical students. The experiment also took place in a controlled lab setting. To ensure that the results apply for a broader population in more general settings, we would need to run a study with more balanced representation from additional groups in uncontrolled everyday environments. Additionally, the single-handed nature of the experiment interactions is somewhat limiting. To gain more understanding of practical human-robot interactions for everyday use, we will need to expand to more diverse and relevant two-handed interactions. Furthermore, we relied on self-reported measures for our affect analysis, rather than assessing engagement-related metrics through more objective data-driven means such as facial expression analysis. Finally, although the experimenter did their best to convey equipoise throughout the study, the within-subjects design may have exaggerated differences between conditions due to demand characteristics.

Our next research steps will address the encountered issues for improved future experimental design and robot motion. Most user confusion and some of the reactivity mode issues could be addressed by giving the user more instruction and practice trials at the beginning of the study. Other improvement steps include defining leadership roles more consistently throughout each trial and modifying robot reaction behavior to allow for constantly-adapting retreat positions. We can rectify the "jazz hands" oscillation issue by redesigning the end-effector to make it more difficult for users to exert axial torque and also re-tuning the controller with increased W2 joint damping. In future experiments, we plan to recruit a more diverse pool of participants and explore additional interaction engagement metrics in both withinsubjects and between-subjects paradigms.

\section{Conclusion}

Overall, we are energized by the positive reactions to this study and are eager to create an improved version of this system. The affective results give us a guide to manipulating the emotional experience of robot users by adjusting known parameters. The disparate effects of facial animation and arm stiffness hold particular potential for changing specific aspects of user experience; experimenters can adjust the pleasantness and energeticness of a robot by altering only the robot's facial reactivity while separately manipulating the robot's safety appearance and dominance by changing the stiffness of passive arm joints. Robot timing had a strong effect on user perceptions of the robot as well. Particularly, a faster interaction tempo can make the robot seem more energetic and more dominant as needed. Now that we know how it feels to clap hands with a robot, this initial human-robot investigation will inform future work on our hand-clapping robotic system in various playful HRI settings. Our findings, especially those elucidating how to tune different interaction parameters to shape an affective HRI experience, may help other researchers in their own spHRI work.

Acknowledgements Open access funding provided by Max Planck Society. The first author was supported by a US National Science Foundation (NSF) Graduate Research Fellowship under Grant No. DGE-0822 and the University of Pennsylvania's NSF Integrative Graduate Education and Research Traineeship under Grant No. 0966142. We thank Michelle Neuburger, a past summer Research Experiences for Undergraduates (REU) student, for her help with this work; she was supported by NSF Grant No. 1156366. We additionally thank Alex Burka for his design advice and Kostas Daniilidis for the use of his Baxter robot. 
Funding This research was funded by the US National Science Foundation (Grant Numbers DGE-0822, 0966142, and 1156366).

\section{Compliance with Ethical Standards}

Conflict of interest Naomi T. Fitter and Katherine J. Kuchenbecker have received research grants from the US National Science Foundation. Naomi T. Fitter now works as an Assistant Professor in the School of Mechanical, Industrial, and Manufacturing Engineering at Oregon State University. She previously worked as a postdoctoral scholar at the University of Southern California. Katherine J. Kuchenbecker now works as a Director at the Max Planck Institute for Intelligent Systems. Katherine J. Kuchenbecker has also served as the Chief Science Advisor for Tactai, Inc. and VerroTouch Medical, Inc.

Open Access This article is distributed under the terms of the Creative Commons Attribution 4.0 International License (http://creativecomm ons.org/licenses/by/4.0/), which permits unrestricted use, distribution, and reproduction in any medium, provided you give appropriate credit to the original author(s) and the source, provide a link to the Creative Commons license, and indicate if changes were made.

\section{References}

1. Ammi M, Demulier V, Caillou S, Gaffary Y, Tsalamlal Y, Martin JC, Tapus A (2015) Haptic human-robot affective interaction in a handshaking social protocol. In: Proceedings ACM/IEEE international conference on human-robot interaction (HRI), pp 263-270

2. Argall BD, Billard AG (2010) A survey of tactile human-robot interactions. Robot Auton Syst 58(10):1159-1176

3. Avraham G, Nisky I, Fernandes HL, Acuna DE, Kording KP, Loeb GE, Karniel A (2012) Toward perceiving robots as humans: three handshake models face the Turing-like handshake test. IEEE Trans Haptics 5(3): 196-207

4. Baba T, Ushiama T, Tsuruno R, Tomimatsu K (2007) Video game that uses skin contact as controller input. In: Proceedings ACM SIGGRAPH Emerging Technologies, p 4

5. Baltrušaitis $T$, Mahmoud $M$, Robinson $P$ (2015) Cross-dataset learning and person-specific normalisation for automatic action unit detection. In: Proceedings IEEE international conference and workshops on automatic face and gesture recognition (FG), vol 6, pp 1-6

6. Bandura A (1992) Social cognitive theory of social referencing. In: Feinman S (ed) Social referencing and the social construction of reality in infancy. Springer, Boston, pp 175-208

7. Bhattacharjee T, Niemeyer G (2015) Antagonistic muscle based robot control for physical interactions. In: Proceedings IEEE international conference on robotics and automation (ICRA), pp 298-303

8. Chan WP, Parker CAC, Van der Loos HFM, Croft EA (2013) A human-inspired object handover controller. Int J Robot Res (IJRR) 32(8):971-983

9. Cooney MD, Becker-Asano C, Kanda T, Alissandrakis A, Ishiguro H (2010) Full-body gesture recognition using inertial sensors for playful interaction with small humanoid robot. In: Proceedings IEEE/RSJ international conference on intelligent robots and systems (IROS), pp 2276-2282

10. DiSalvo CF, Gemperle F, Forlizzi J, Kiesler S (2002) All robots are not created equal: the design and perception of humanoid robot heads. In: Proceedings ACM conference on designing interactive systems, pp 321-326
11. Fiene JP, Kuchenbecker KJ (2007) Shaping event-based haptic transients via an improved understanding of real contact dynamics. In: Proceedings IEEE world haptics conference, pp 170-175

12. Fitter NT, Hawkes DT, Johnson MJ, Kuchenbecker KJ (2016) Designing human-robot exercise games for Baxter. In: Proceedings IEEE/RSJ international conference on intelligent robots and systems (IROS), work in progress, pp 3434-3435

13. Fitter NT, Huang YE, Mayer JP, Kuchenbecker KJ (2016) IMUmediated real-time human-Baxter hand-clapping interaction. In: Proceedings IEEE/RSJ international conference on intelligent robots and systems (IROS), work in progress, pp 3438-3439

14. Fitter NT, Kuchenbecker KJ (2016) Designing and assessing expressive open-source faces for the Baxter robot. In: Proceedings international conference on social robotics (ICSR), pp 340-350

15. Fitter NT, Kuchenbecker KJ (2016) Equipping the Baxter robot with human-inspired hand-clapping skills. In: Proceedings IEEE international symposium on robot and human interactive communication (RO-MAN), pp 105-112

16. Fitter NT, Kuchenbecker KJ (2016) Qualitative user reactions to a hand-clapping humanoid robot. In: Proceedings international conference on social robotics (ICSR), pp 317-327

17. Heerink M, Krose B, Evers V, Wielinga B (2009) Measuring acceptance of an assistive social robot: a suggested toolkit. In: Proceedings IEEE international symposium on robot and human interactive communication (RO-MAN), pp 528-533

18. Huber M, Rickert M, Knoll A, Brandt T, Glasauer S (2008) Humanrobot interaction in handing-over tasks. In: Proceedings IEEE international symposium on robot and human interactive communication (RO-MAN), pp 107-112

19. Iwata $H$, Sugano $S$ (2005) Human-robot-contact-state identification based on tactile recognition. IEEE Trans Ind Electron 52(6):14681477

20. Kanda T, Sato R, Saiwaki N, Ishiguro H (2007) A two-month field trial in an elementary school for long-term human-robot interaction. IEEE Trans Robot 23(5):962-971

21. Kim Y, Lee S, Hwang I, Ro H, Lee Y, Mo on, M, Song J (2014) High5: promoting interpersonal hand-to-hand touch for vibrant workplace with electrodermal sensor watches. In: Proceedings $\mathrm{ACM}$ international joint conference on pervasive and ubiquitous computing (UbiComp), pp 15-19

22. Klemmer SR, Hartmann B, Takayama L (2006) How bodies matter: five themes for interaction design. In: Proceedings ACM conference on designing interactive systems, pp 140-149

23. Mehrabian A (1980) Basic dimensions for a general psychological theory. Oelgeschlager, Gunn \& Hain, Cambridge

24. Noy L, Dekel E, Alon U (2011) The mirror game as a paradigm for studying the dynamics of two people improvising motion together. Proc Natl Acad Sci 108(52):20947-20952

25. Rabbitt SM, Kazdin AE, Scassellati B (2015) Integrating socially assistive robotics into mental healthcare interventions: applications and recommendations for expanded use. Clin Psychol Rev 35:3546

26. Robins B, Dautenhahn K, Te Boekhorst R, Billard A (2005) Robotic assistants in therapy and education of children with autism: can a small humanoid robot help encourage social interaction skills? Univers Access Inf Soc 4(2): 105-120

27. Schmidt RC, Fitzpatrick P, Caron R, Mergeche J (2011) Understanding social motor coordination. Hum Mov Sci 30(5):834-845

28. Shibata T, Tanie K (2001) Physical and affective interaction between human and mental commit robot. In: Proceedings IEEE international conference on robotics and automation (ICRA), vol 3, pp 2572-2577

29. Sonneveld MH, Schifferstein HNJ (2008) The tactual experience of objects. In: Product Experience, pp 41-67

30. Stiehl WD, Breazeal C, Han KH, Lieberman J, Lalla L, Maymin A, Salinas J, Fuentes D, Toscano R, Tong CH, et al (2006) The 
Huggable: a therapeutic robotic companion for relational, affective touch. In: Proceedings ACM SIGGRAPH conference, emerging technologies, p 15

31. Visell Y, Okamoto S (2014) Vibrotactile sensation and softness perception. In: Di Luca M (ed) Multisensory softness. Springer, London, pp 31-47

32. Weiss A, Bernhaupt R, Tscheligi M, Wollherr D, Kuhnlenz K, Buss M (2008) A methodological variation for acceptance evaluation of human-robot interaction in public places. In: Proceedings IEEE international symposium on robot and human interactive communication (RO-MAN), pp 713-718

33. Yohanan S, MacLean K (2009) A tool to study affective touch: goals \& design of the haptic creature. In: Proceedings ACM CHI conference on human factors in computing systems (CHI), spotlight on works in progress, pp 4153-4158

34. Yohanan S, MacLean KE (2012) The role of affective touch in human-robot interaction: human intent and expectations in touching the haptic creature. Int J Soc Robot (SORO) 4(2):163-180

35. Zhou A, Hadfield-Menell D, Nagabandi A, Dragan AD (2017) Expressive robot motion timing. In: Proceedings ACM/IEEE international conference on human-robot interaction (HRI), pp 22-31

Publisher's Note Springer Nature remains neutral with regard to jurisdictional claims in published maps and institutional affiliations.

Naomi T. Fitter is an is an Assistant Professor in the School of Mechanical, Industrial, and Manufacturing Engineering at Oregon State University. Her past degrees include a B.S. and B.A. in mechanical engineering and Spanish from the University of Cincinnati and an M.S.E. and Ph.D. in Robotics and Mechanical Engineering and Applied Mechanics from the University of Pennsylvania. She completed her doctoral work in the GRASP Laboratory and did postdoctoral research at the University of Southern California. Her research aims to equip robots with the ability to engage and empower people in interactions from playful high-fives to challenging physical therapy routines.
Katherine J. Kuchenbecker directs the Haptic Intelligence Department at the Max Planck Institute for Intelligent Systems in Stuttgart, Germany. She earned her Ph.D. in Mechanical Engineering at Stanford University in 2006, did postdoctoral research at the Johns Hopkins University, and was an engineering professor at the University of Pennsylvania before she moved to the Max Planck Society in 2017. Her research centers on haptic interfaces, which enable a user to touch virtual and distant objects as though they were real and within reach, as well as haptic sensing systems, which allow robots to physically interact with objects and people. She delivered a TEDYouth talk on haptics in 2012 and has been honored with a 2009 NSF CAREER Award, the 2012 IEEE Robotics and Automation Society Academic Early Career Award, a 2014 Penn Lindback Award for Distinguished Teaching, and various best paper and best demonstration awards. She co-chaired the IEEE Technical Committee on Haptics from 2015 to 2017 and co-chaired the IEEE Haptics Symposium in 2016 and 2018. 